\title{
Attention, please!
}

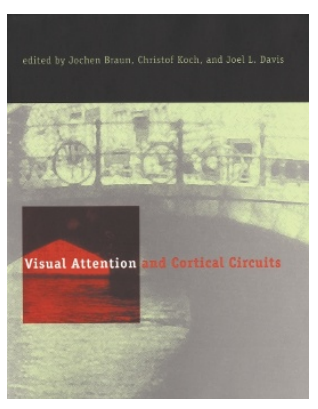

\section{Visual Attention and Cortical Circuits}

edited by Jochen Braun, Christof Koch and Joel L. Davis

MIT Press, Cambridge, Massachusetts, 2001. \$48

hardcover, pp 344

ISBN 0-26-202493-4

Reviewed by Sabine Kastner

Our senses are typically bombarded with a vast amount of information that cannot all be processed simultaneously in the nervous system. Selective attention helps us to handle the processing of information from cluttered sensory environments by selecting relevant and by filtering out irrelevant stimuli. The importance of attentional mechanisms for our everyday life becomes immediately obvious when they fail, as in patients with attentional deficits, such as children suffering from ADHD. The study of attention is a major topic in many different disciplines, such as cognitive psychology, neurobiology and computational neuroscience, which use a variety of methodological approaches. Psychologists typically investigate the effects of attention by using behavioral performance measures. For example, selective attention to a spatial location improves the accuracy and speed of responses to stimuli that occur in that location. Physiologists study neural correlates of attention in different parts of the brain using single-cell recordings in monkeys or functional brain mapping in humans. One of the major findings has been that neural responses to an attended sensory stimulus are enhanced in sensory representation areas compared to the responses evoked by the same stimulus when unattended. Because of the different expertise and approaches of attention researchers, it has been proven difficult to derive a unified framework of selective attention that can account for the majority of empirical findings from psychology and neurobiology. A major goal of cognitive neuroscience is to bridge multiple disciplines by correlating behavioral and neural measures in order to develop an integrated theory of attention.

Sabine Kastner is in the Department of

Psychology, Center for the Study of Brain, Mind, and Behavior, Princeton University, Princeton,

New Jersey 08540, USA.

e-mail: skastner@princeton.edu
Visual Attention and Cortical Circuits is aimed at the development of such an integrated theory of attention; the volume presents the results of a workshop held in early 1999 that brought together prominent attention researchers from psychology, neurobiology and computational neuroscience. The goal of the workshop was to enlarge the 'common ground' among these disciplines and to find a common language that would enable them to learn from one another. The book focuses mainly on the effects of visual attention on ventral and dorsal stream areas in human and monkey visual cortex, and corresponding changes in behavioral performance. Although the contributors did not span all areas of current attention research, the major conceptual and methodological approaches in the field are well represented.

The fourteen chapters cover a wide range of topics. Several contributions are concerned with the modulation of sensory-evoked neural activity by selective attention in visual cortex. Such attentional modulation has been shown at all levels of visual processing, including V1 in the monkey and human visual systems. Another topic covered by several authors concerns mechanisms of attentional control, with particular emphasis on frontal lobe function. Each chapter exemplifies the approach of a particular group of scientists to the study of attention, and there is some overlap among chapters. Thereby, the reader gains fascinating insights into the toolbox of attention researchers who use different techniques such as functional brain mapping, psychophysics, behavioral studies in patients with focal brain lesions, single-cell physiology, and combinations of these to study similar themes. The book critically discusses the difficulties in combining different methodological approaches; however, such an interdisciplinary approach to the study of atten- tion as well as to other research areas seems to be invaluable. The chapters are generally well written and provide comprehensive overviews of previous and current research of the particular groups. Because of the 'bicultural approach' in combining psychophysical measures with single-unit physiology, my favorites were the chapters by Thompson and colleagues on attentional function of the monkey frontal eye fields and by Ito and colleagues on attentional modulation in primary visual cortex. I also found the chapters on theoretical concepts of selective attention particularly strong. Originally, these theories attempted to formally describe behavioral findings, but they now encompass increasing numbers of neurobiological findings. The individual contributions of the volume represent discrete units and do not depend on one another, which minimizes redundancy and overlap among them. However, on the downside, it is not often apparent how the individual contributions fit into a larger framework of selective attention that integrates behavioral and neural measures. In most of the chapters, there are too few cross-references to the other contributions; a brief discussion of the individual findings in light of a larger picture at the end of each chapter would have been helpful for readers from outside the attention field. However, the editors attempted to integrate the individual chapters in an introductory overview by summarizing and highlighting the main findings of each contribution, emphasizing the larger points that emerge from the volume as a whole. This summary is valuable in pointing out some of the most important current questions in the field of attention and by attempting to integrate the findings from psychophysics, neurobiology and computational theory into a comprehensive framework of visual attention.

The editors' goal was to "derive a comprehensive theory of attention from both neurobiological and psychological data." As a result, the current volume is an important first of many steps in the pursuit of a unified theoretical framework for visual attention. It will hopefully stimulate more interdisciplinary communication and collaboration, which will be key in advancing our knowledge in the attention field and other areas of neuroscience. The book is highly recommended for advanced students and researchers from a variety of fields, such as neurology, neurobiology, psychology and computer vision, with a particular interest in attention research. 